\title{
Monitoring the Extragalactic High Energy Sky
}

\author{
Jean-Philippe Lenain ${ }^{+}(1)$ \\ Sorbonne Université, Université Paris Diderot, Sorbonne Paris Cité, CNRS/IN2P3, Laboratoire de Physique \\ Nucléaire et de Hautes Energies, LPNHE, 4 Place Jussieu, F-75252 Paris, France; \\ contact.hess@hess-experiment.eu \\ + On behalf of the H.E.S.S. Collaboration.
}

Received: 27 November 2018; Accepted: 23 December 2018; Published: 3 January 2019

\begin{abstract}
Blazars are jetted active galactic nuclei with a jet pointing close to the line of sight, hence enhancing their intrinsic luminosity and variability. Monitoring these sources is essential in order to catch them flaring and promptly organize follow-up multi-wavelength observations, which are key to providing rich data sets used to derive e.g., the emission mechanisms at work, and the size and location of the flaring zone. In this context, the Fermi-LAT has proven to be an invaluable instrument, whose data are used to trigger many follow-up observations at high and very high energies. A few examples are illustrated here, as well as a description of different data products and pipelines, with a focus given on FLaapLUC, a tool in use within the H.E.S.S. collaboration.
\end{abstract}

Keywords: active galactic nuclei; blazars; gamma rays; high energy; monitoring

\section{Introduction}

Active galactic nuclei (AGN) are among the most energetic sources in the Universe. They harbor a supermassive black hole in the center of their host galaxy, fueled by an accretion disk emitting mainly UV and X-ray thermal radiation. Blazars are a specific class of AGN, exhibiting relativistic jets, with emission dominated by non-thermal radiation from the radio to the $\gamma$-ray bands. The observational peculiarity of blazars stems from the quasi-alignment of their jet with our line of sight, thus Doppler boosting their emission [1,2]. Thus, their luminosity can be highly variable in almost all wavebands, and this variability can occur on a broad range of time scales, from years down to minutes.

Blazars are further divided in sub-classes: flat spectrum radio quasars (FSRQ), low-frequency-peaked BL Lac objects (LBL), intermediate-frequency-peaked BL Lac objects (IBL) and high-frequency-peaked BL Lac objects (HBL), depending on the peak frequency of their synchrotron component, but which also display different luminosities. FSRQ exhibit emission lines in the optical/UV range from e.g., their accretion disk, while BL Lac objects are mostly devoid of any detectable thermal component. From these different types, Fossati et al. [3] proposed the existence of a blazar sequence, characterized by an anti-correlation between peak frequency and luminosity. Since then, several studies have been conducted to assess whether this sequence is real or due to selection effects (see, e.g., [4-9]). In the meantime, several outliers from this sequence have been detected, with, for instance, objects exhibiting both a high peak frequency and bolometric luminosity (e.g., $[10,11])$. This subject is still a matter of debate (see, e.g., [12,13], and references therein).

The spectral energy distribution (SED) of blazars typically shows two main components (see, e.g., Figure 1). A low-energy component, peaking in the infrared to X-ray band, is commonly ascribed to synchrotron emission from relativistic electrons and positrons in the jet. The nature of the high energy component, peaking at high energies, can depend on the source properties and/or activity state. Leptons from the jet can interact with their own synchrotron emission by inverse Compton scattering, via the synchrotron self-Compton mechanism [14,15]. In FRSQ or LBL objects, the thermal emission 
from the accretion disk, from the broad line region or the dusty torus can contribute significantly and give rise to external inverse Compton emission at high energies. In a hadronic framework, proton synchrotron and $p-\gamma$ interactions can dominate the high energy emission (see, e.g., $[16,17])$.

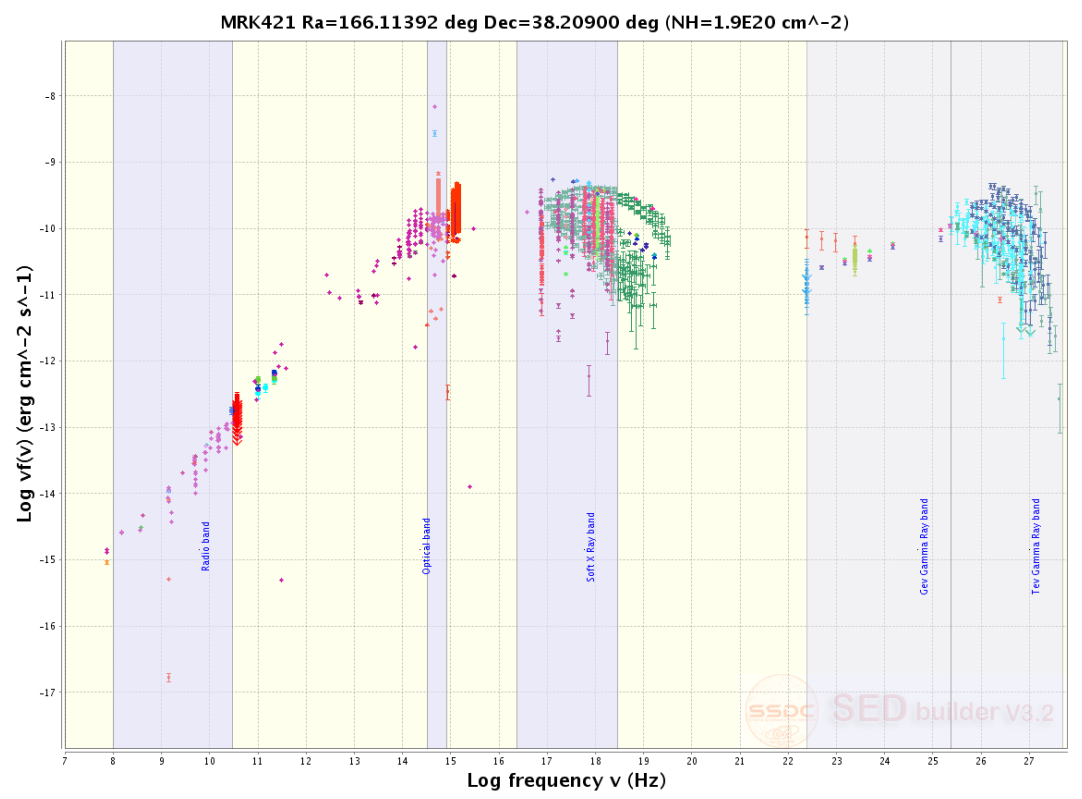

Figure 1. Spectral energy distribution of Mrk 421, showing the two typical spectral components, and variability, generated using https:/ / tools.ssdc.asi.it/SED, data from [18-60].

The study of the variability in blazars, essentially of their flares, can help to reveal the nature of the emission mechanisms, as well as the location of the source emission (e.g., [61-63]). By contrast, in order to detect the thermal emission, investigable mostly in the near-infrared/optical range, the sources should be caught while in a quiet non-thermal state, such that the usually dominating non-thermal component does not outshine the thermal one. This is an almost necessary condition to reliably measure their redshift, obviously an essential quantity to derive the energetic budget in these objects (see, e.g., $[64,65])$.

\section{Facilities and Coverage}

Catching AGN in flaring state, or quieter activity state for redshift measurements for instance, requires monitoring them with high cadence, given the diverse variability time scales they display. However, most astrophysical facilities have small fields of view, with respect to the entire sky, and rare are the instruments capable of instantly observing a sizable fraction of the celestial vault. Furthermore, depending on the observed wavelength and modes of detection, most instruments can not continuously operate. Such low duty cycles and small fields of view make exhaustive monitoring programs difficult to achieve, either by selecting a few sources on which observational efforts are focused, or due to high time pressure on the overall facility's observation program. In a few cases, it is possible to gather different observatories to observe the same source at almost the same time (see, e.g., Figure 2), at the expense of notable efforts. This has been brilliantly demonstrated very recently during the multi-wavelength and multi-messenger campaign on NGC 4993 at the occasion of the binary neutron star merger event GW 170817 [66], or for the campaign organized on the presumable electromagnetic counterpart TXS 0506+056 of the neutrino event IceCube-170922A [67]. However, observations performed with facilities having small fields of view and/or low duty cycle tend to be naturally biased to observations of high-flux states, if no long-term, persevering monitoring programs with regular cadence are in place. 


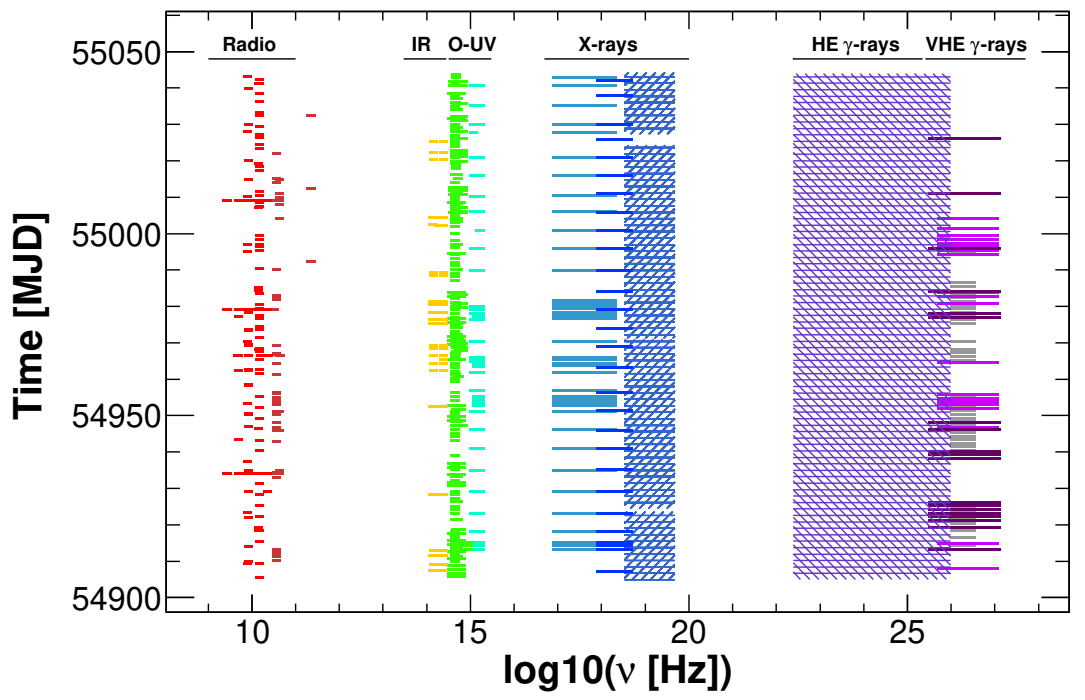

Figure 2. Time and energy band coverage of the multi-wavelength campaign on Mrk 501 in 2009, from Abdo et al. [68].

On the opposite scale, some facilities such as the Neil Gehrels Swift/BAT [69], AGILE [70], Fermi-GBM [71] and Fermi-LAT [72] or HAWC [73] have all-sky capabilities, with a large field of view, and almost continuous or high cadence observations (see Figure 3). Without such monitoring capabilities, it would have been, for instance, barely possible to serendipitously discover flares from the Crab Nebula, previously thought to be a steady $\gamma$-ray source (see, e.g., [74], and references therein). Furthermore, at high Galactic latitudes, the high energy $\gamma$-ray sky is largely dominated by AGN, and particularly blazars [56], making AGILE and Fermi-LAT perfect instruments to monitor the high energy extragalactic sky.
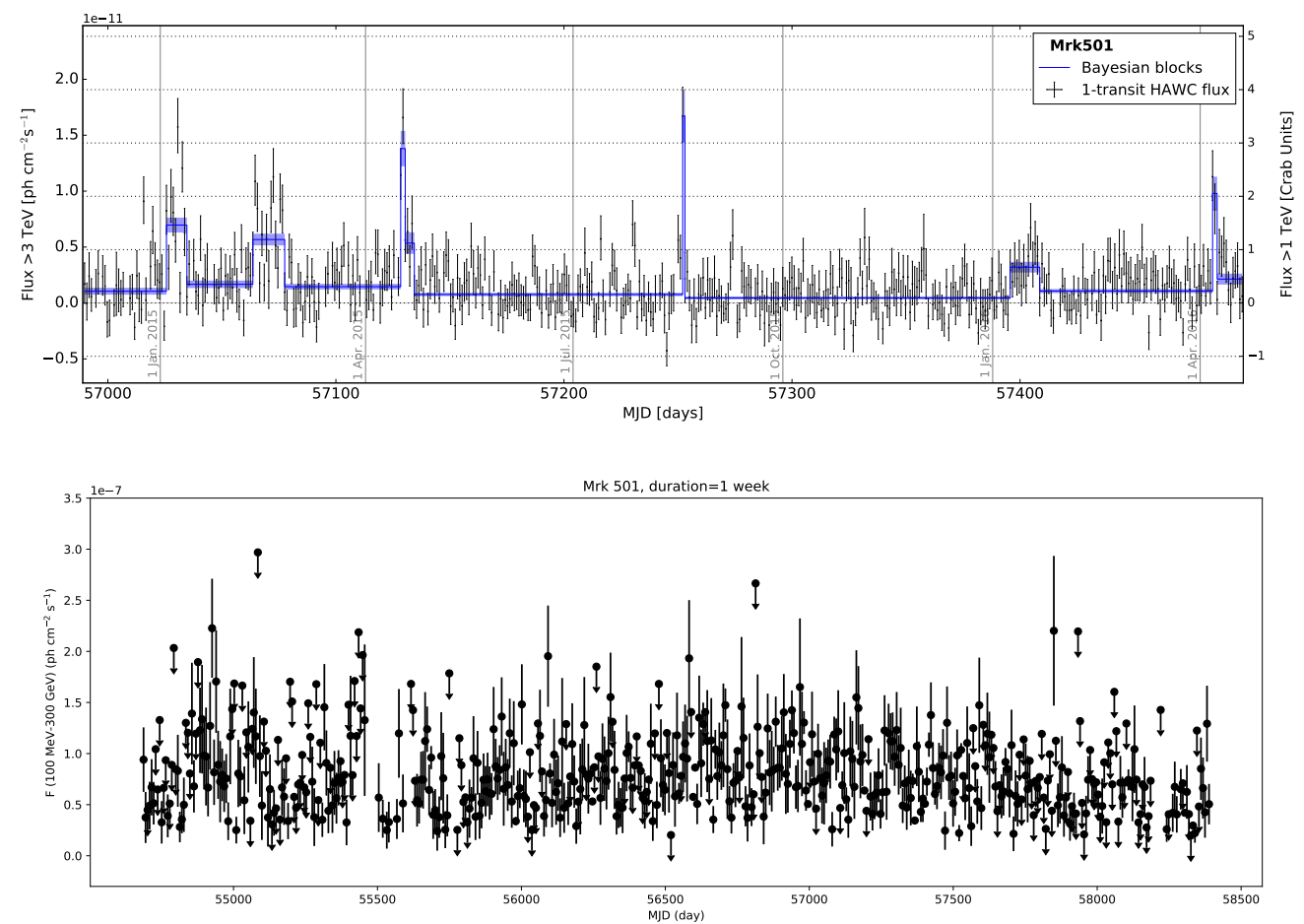

Figure 3. (Top) Light curve of Mrk 501 as seen with HAWC from November 2014 to April 2016, with a daily sampling. The blue lines show distinct flux states from a Bayesian block analysis, from Abeysekara et al. [75]; (Bottom) long-term, weekly-binned light curve of Mrk501, as observed with Fermi-LAT for 10 years. Adapted from [76]. 
In particular, the Fermi-LAT has proven to be very useful to alert the community on unusual events for follow-up observations. As examples, observations at very high energies (VHE; $E \gtrsim 100 \mathrm{GeV}$ ) following up source activity detected at high energies with the Fermi-LAT yielded the following recent results:

- the detection of the FSRQ Ton 599 by both MAGIC [77] and VERITAS [78], following an alert from Fermi-LAT [79];

- the discovery of VHE emission from a gravitationally lensed blazar, S30218+357, at the time of arrival of the lensed component $[80,81]$, which followed the prompt flare reported by Fermi-LAT [82], making it the furthest VHE $\gamma$-ray source known to date with $z=0.944$;

- the detection of the second furthest VHE $\gamma$-ray source (with $z=0.939$ ), the FSRQ PKS $1441+25$, with MAGIC [83] and VERITAS [84] following activity detected in the optical, X-ray and high-energy $\gamma$-ray ranges [85];

- the detection at VHE of the FSRQ PKS 0736+017 with H.E.S.S.-Ref. [86] following a $\gamma$-ray flare identified as explained in the following.

\section{Fermi-LAT as an All-Sky Monitor and Flare Detector}

The Fermi-LAT team and the Fermi Science Support Center (FSSC) at NASA provide numerous data products and analysis tools to the community, from the Fermitools ${ }^{1}$ needed to analyze data, to high-level data products ${ }^{2}$. Catalog products, such as those for the 3FGL [56] or 3FHL [87], are also made available.

Of interest for source monitoring and potential follow-up observations on relevant source activities are the available light curves for every source having experienced a flaring activity with a flux exceeding $10^{-6} \mathrm{~cm}^{-2} \mathrm{~s}^{-1}$ at least once during the Fermi-LAT mission lifetime ${ }^{3}$, as well as the light curves obtained using aperture photometry of all sources belonging to the 3FGL catalog ${ }^{4}$. It should be noted, though, that these light curves have no absolute flux calibration but are relevant to reveal changes of activity, i.e., relative flux variations, from a given object. Another interesting product is given by the Fermi All-sky Variability Analysis (FAVA) tool ${ }^{5}[88,89]$, which blindly searches the whole sky to detect flares from $\gamma$-ray sources. One caveat related to the use of this tool is that its latency for flare detection is of the order of a week in order to accumulate enough statistics, which is sufficient for long-lasting events, but too long for e.g., some AGN flares which may last only a few days or less.

As a result, one may be interested in piping such products into an automatic stream such that follow-up observations could be promptly organized, or one may want to monitor other sources not covered by the aforementioned products, or to keep control on flux thresholds on which follow-up alerts should be issued. In that spirit, it is worth noting that Errando and Orr [90] developed pipelines digesting Fermi-LAT data for potential target of opportunity (ToO) observations with VERITAS. We give another example below, which is in use in the H.E.S.S. collaboration.

Within the H.E.S.S. collaboration, the tool FLaapLUC (Eermi-LAT automatic aperture photometry Light $\underline{\underline{C}} \leftrightarrow$ Urve) has been developed to identify flares in Fermi-LAT data [91]. The purpose is to catch unusual activity in high-energy emitting sources as quickly as possible using Fermi-LAT data, while the flare is still on the go, in order to promptly organize ToO observations with H.E.S.S. The method used here is also based on the aperture photometry ${ }^{6}$, which is computationally fast but inaccurate in terms of absolute flux scale, as mentioned above.

\footnotetext{
https://fermi.gsfc.nasa.gov/ssc/data/analysis/software

https://fermi.gsfc.nasa.gov/ssc/data/access/lat

https://fermi.gsfc.nasa.gov/ssc/data/access/lat/msl_lc

https://fermi.gsfc.nasa.gov/ssc/data/access/lat/4yr_catalog/ap_lcs.php

https: / / fermi.gsfc.nasa.gov/ssc/data/access/lat/FAVA

https://fermi.gsfc.nasa.gov/ssc/data/analysis/scitools/aperture_photometry.html
} 
More than 320 AGN are monitored daily with FLaapLUC. This tool takes advantage of the whole mission data set of Fermi-LAT to construct a long-term average flux $\left(\overline{F_{\mathrm{LT}}}\right)$ for each source, which serves as the baseline for the identification of flares. Typically, if the last daily flux measurement of a source is above $\overline{F_{\mathrm{LT}}}+3 \cdot \operatorname{RMS}\left(F_{\mathrm{LT}}\right)$ (see Figure 4 ), then an automatic alert is issued. In that case, a follow-up likelihood analysis is automatically processed to derive a precise spectral characterization. This alert is handled within the H.E.S.S. AGN ToO group, which then evaluates the feasibility, and potentially triggers follow-up ToO observations with H.E.S.S. FLaapLUC veto alerts depending on the source distance and zenith angle at culmination, to take into account $\gamma$-ray absorption by the extragalactic background light [92], as well as to account for source visibility at the H.E.S.S. site. For more details on FLaapLUC, whose code is available at https:/ / github.com/jlenain/flaapluc, the reader is invited to Lenain [91]. FLaapLUC has been in use in H.E.S.S. since late 2012. Recently, H.E.S.S. detected VHE flares following observations triggered with FLaapLUC, for instance from 3C 279 [93], TXS 0506+056 along with a Fermi-LAT alert [94], PKS 2022-07, PKS 1749+096 or PKS 0736+017 [95,96].

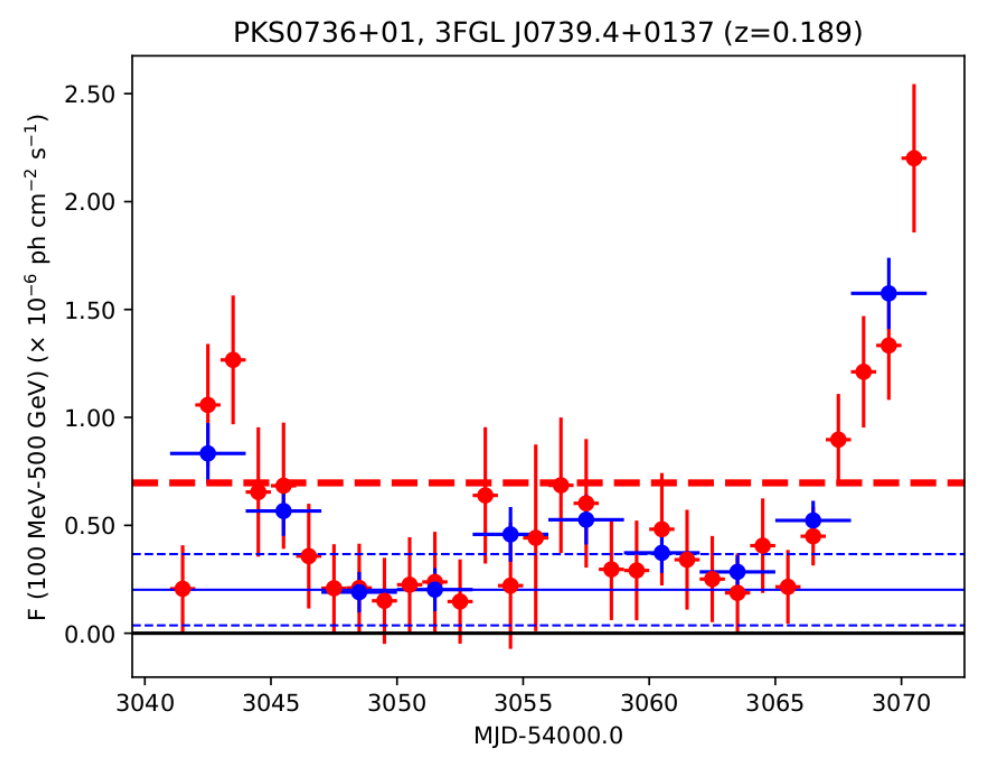

Figure 4. One month segment of the Fermi-LAT light curve from PKS 0736+01 from FLaapLUC. H.E.S.S. observations were triggered and observations begun following the last measurement. The blue points show the data with bins of three days, while the red points represent the daily-binned data set. The solid blue line shows the long-term (10 years) flux average with $1 \sigma$ fluctuations (dashed blue lines), and the dashed red line shows the flux alert threshold for this source.

\section{Conclusions}

Monitoring the high-energy sky is key for making new discoveries, either of previously unknown events, such as for the presumable coincidence of $\gamma$ rays with neutrinos as observed in TXS 0506+056 [67], or to follow up on flaring events in known AGN. To do so, all-sky or large field-of-view instruments, with high duty cycle, such as HAWC or Fermi-LAT, are invaluable. Long-term monitoring programs as a strategy for observations, such as those performed by the FACT telescope [97], are also a key asset, since they provide regular observations of selected sources in an unbiased way [98].

In the near future, the future CTA (Cherenkov Telescope Array) observatory is expected to provide, as parts of its key science projects, long-term monitoring of selected AGN, as well as a survey of a part of the extragalactic sky and of the Galactic plane (see [99], for more details). Given its field of view of the order of a few degrees, CTA will presumably provide a shallow and wide survey of $\sim 25 \%$ of the sky, with an expected sensitivity of more than ten times better than Fermi-LAT or HAWC for 
steady sources in its core energy range $(100 \mathrm{GeV}-10 \mathrm{TeV})$. This survey is expected to be completed within the first few years of operation of CTA, and thus complementary survey-capable facilities, such as e-ASTROGAM [100] or $\mathrm{AMEGO}^{7}$, are necessary to take such a survey over in the next couple of decades. Such satellite projects being still in the proposal stage, assuring that Fermi keeps flying in the next years is essential for the community.

Funding: This research received no external funding.

Acknowledgments: The support of the Namibian authorities and of the University of Namibia in facilitating the construction and operation of H.E.S.S. is gratefully acknowledged, as is the support by the German Ministry for Education and Research (BMBF), the Max Planck Society, the German Research Foundation (DFG), the Helmholtz Association, the Alexander von Humboldt Foundation, the French Ministry of Higher Education, Research and Innovation, the Centre National de la Recherche Scientifique (CNRS/IN2P3 and CNRS/INSU), the Commissariat à l'énergie atomique et aux énergies alternatives (CEA), the U.K. Science and Technology Facilities Council (STFC), the Knut and Alice Wallenberg Foundation, the National Science Centre, Poland Grant No. 2016/22/M/ST9/00382, the South African Department of Science and Technology and National Research Foundation, the University of Namibia, the National Commission on Research, Science \& Technology of Namibia (NCRST), the Austrian Federal Ministry of Education, Science and Research and the Austrian Science Fund (FWF), the Australian Research Council (ARC), the Japan Society for the Promotion of Science and by the University of Amsterdam. We appreciate the excellent work of the technical support staff in Berlin, Zeuthen, Heidelberg, Palaiseau, Paris, Saclay, Tübingen and in Namibia in the construction and operation of the equipment. This work benefitted from services provided by the H.E.S.S. Virtual Organisation, supported by the national resource providers of the EGI Federation.

This research has made use of NASA's Astrophysics Data System, of the SIMBAD database, operated at CDS, Strasbourg, France, and of the TeVCat online source catalog (http:// tevcat.uchicago.edu). Part of this work is based on archival data, software or online services provided by the Space Science Data Center-ASI.

Conflicts of Interest: The author declares no conflict of interest.

\section{References}

1. Rees, M.J. Appearance of Relativistically Expanding Radio Sources. Nature 1966, 211, 468-470, doi:10.1038/211468a0. [CrossRef]

2. Blandford, R.D.; Rees, M.J. Extended and compact extragalactic radio sources-Interpretation and theory. Phys. Scr. 1978, 17, 265-274. [CrossRef]

3. Fossati, G.; Maraschi, L.; Celotti, A.; Comastri, A.; Ghisellini, G. A unifying view of the spectral energy distributions of blazars. Mon. Not. R. Astron. Soc. 1998, 299, 433-448. [CrossRef]

4. Padovani, P. The blazar sequence: Validity and predictions. Astrophys. Space Sci. 2007, 309, 63-71, doi:10.1007/s10509-007-9455-2. [CrossRef]

5. Nieppola, E.; Valtaoja, E.; Tornikoski, M.; Hovatta, T.; Kotiranta, M. Blazar sequence—An artefact of Doppler boosting. Astron. Astrophys. 2008, 488, 867-872, doi:10.1051/0004-6361:200809716. [CrossRef]

6. Ghisellini, G.; Tavecchio, F. The blazar sequence: A new perspective. Mon. Not. R. Astron. Soc. 2008, 387, 1669-1680, doi:10.1111/j.1365-2966.2008.13360.x. [CrossRef]

7. Maraschi, L.; Foschini, L.; Ghisellini, G.; Tavecchio, F.; Sambruna, R.M. Testing the blazar spectral sequence: X-ray-selected blazars. Mon. Not. R. Astron. Soc. 2008, 391, 1981-1993, doi:10.1111/j.1365-2966.2008.14030.x. [CrossRef]

8. Meyer, E.T.; Fossati, G.; Georganopoulos, M.; Lister, M.L. From the Blazar Sequence to the Blazar Envelope: Revisiting the Relativistic Jet Dichotomy in Radio-loud Active Galactic Nuclei. Astrophys. J. 2011, 740, 98, doi:10.1088/0004-637X/740/2/98. [CrossRef]

9. Giommi, P.; Padovani, P.; Polenta, G.; Turriziani, S.; D’Elia, V.; Piranomonte, S. A simplified view of blazars: Clearing the fog around long-standing selection effects. Mon. Not. R. Astron. Soc. 2012, 420, 2899-2911, doi:10.1111/j.1365-2966.2011.20044.x. [CrossRef]

10. Padovani, P.; Giommi, P.; Rau, A. The discovery of high-power high synchrotron peak blazars. Mon. Not. R. Astron. Soc. 2012, 422, L48, doi:10.1111/j.1745-3933.2012.01234.x. [CrossRef]

7 See https://asd.gsfc.nasa.gov/amego 
11. Cerruti, M.; Benbow, W.; Chen, X.; Dumm, J.P.; Fortson, L.F.; Shahinyan, K. Luminous and high-frequency peaked blazars: The origin of the $\gamma$-ray emission from PKS 1424+240. Astron. Astrophys. 2017, 606, A68, doi:10.1051/0004-6361/201730799. [CrossRef]

12. Ghisellini, G.; Righi, C.; Costamante, L.; Tavecchio, F. The Fermi blazar sequence. Mon. Not. R. Astron. Soc. 2017, 469, 255-266, doi:10.1093/mnras/stx806. [CrossRef]

13. Padovani, P.; Alexander, D.M.; Assef, R.J.; De Marco, B.; Giommi, P.; Hickox, R.C.; Richards, G.T.; Smolčić, V.; Hatziminaoglou, E.; Mainieri, V.; et al. Active galactic nuclei: What's in a name? Astron. Astrophys. Rev. 2017, 25, 2, doi:10.1007/s00159-017-0102-9. [CrossRef]

14. Ginzburg, V.L.; Syrovatskii, S.I. Cosmic Magnetobremsstrahlung (synchrotron Radiation). Annu. Rev. Astron. Astrophys. 1965, 3, 297, doi:10.1146/annurev.aa.03.090165.001501. [CrossRef]

15. Konigl, A. Relativistic jets as X-ray and gamma-ray sources. Astrophys. J. 1981, 243, 700-709, doi:10.1086/158638. [CrossRef]

16. Mannheim, K.; Biermann, P.L.; Kruells, W.M. A novel mechanism for nonthermal X-ray emission. Astron. Astrophys. 1991, 251, 723-731.

17. Mücke, A.; Protheroe, R.J. A proton synchrotron blazar model for flaring in Markarian 501. Astropart. Phys. 2001, 15, 121-136, doi:10.1016/S0927-6505(00)00141-9. [CrossRef]

18. Dixon, R.S. A Master List of Radio Sources. Astrophys. J. Suppl. Ser. 1970, 20, 1-503, doi:10.1086/190216. [CrossRef]

19. Warwick, R.S.; Marshall, N.; Fraser, G.W.; Watson, M.G.; Lawrence, A.; Page, C.G.; Pounds, K.A.; Ricketts, M.J.; Sims, M.R.; Smith, A. The Ariel V/3 A/catalogue of X-ray sources-I. Sources at low galactic latitude/absolute value of B less than $10 \mathrm{deg}$. Mon. Not. R. Astron. Soc. 1981, 197, 865-891, doi:10.1093/mnras/197.4.865. [CrossRef]

20. White, R.L.; Becker, R.H. A new catalog of 30,239 1.4 GHz sources. Astrophys. J. Suppl. Ser. 1992, 79, 331-467, doi:10.1086/191656. [CrossRef]

21. Elvis, M.; Plummer, D.; Schachter, J.; Fabbiano, G. The Einstein Slew Survey. Astrophys. J. Suppl. Ser. 1992, 80, 257-303, doi:10.1086/191665. [CrossRef]

22. Gregory, P.C.; Scott, W.K.; Douglas, K.; Condon, J.J. The GB6 Catalog of Radio Sources. Astrophys. J. Suppl. Ser. 1996, 103, 427, doi:10.1086/192282. [CrossRef]

23. White, R.L.; Becker, R.H.; Helfand, D.J.; Gregg, M.D. A Catalog of 1.4 GHz Radio Sources from the FIRST Survey. Astrophys. J. 1997, 475, 479-493, doi:10.1086/303564. [CrossRef]

24. Condon, J.J.; Cotton, W.D.; Greisen, E.W.; Yin, Q.F.; Perley, R.A.; Taylor, G.B.; Broderick, J.J. The NRAO VLA Sky Survey. Astron. J. 1998, 115, 1693-1716, doi:10.1086/300337. [CrossRef]

25. Voges, W.; Aschenbach, B.; Boller, T.; Bräuninger, H.; Briel, U.; Burkert, W.; Dennerl, K.; Englhauser, J.; Gruber, R.; Haberl, F.; et al. The ROSAT all-sky survey bright source catalogue. Astron. Astrophys. 1999, 349, 389-405,

26. Hartman, R.C.; Bertsch, D.L.; Bloom, S.D.; Chen, A.W.; Deines-Jones, P.; Esposito, J.A.; Fichtel, C.E.; Friedlander, D.P.; Hunter, S.D.; McDonald, L.M.; et al. The Third EGRET Catalog of High-Energy Gamma-Ray Sources. Astrophys. J. Suppl. Ser. 1999, 123, 79-202, doi:10.1086/313231. [CrossRef]

27. Giommi, P.; Capalbi, M.; Fiocchi, M.; Memola, E.; Perri, M.; Piranomonte, S.; Rebecchi, S.; Massaro, E. A Catalog of 157 X-ray Spectra and 84 Spectral Energy Distributions of Blazars Observed with BeppoSAX. In Blazar Astrophysics with BeppoSAX and Other Observatories; Giommi, P.; Massaro, E.; Palumbo, G., Eds.; Agenzia Spaziale Ialiana: Rome, Italy, 2002; p. 63.

28. Amenomori, M.; Ayabe, S.; Cui, S.W.; Danzengluobu.; Ding, L.K.; Ding, X.H.; Feng, C.F.; Feng, Z.Y.; Gao, X.Y.; Geng, Q.X.; et al. Multi-TeV Gamma-Ray Flares from Markarian 421 in 2000 and 2001 Observed with the Tibet Air Shower Array. Astrophys. J. 2003, 598, 242-249, doi:10.1086/378350. [CrossRef]

29. Myers, S.T.; Jackson, N.J.; Browne, I.W.A.; de Bruyn, A.G.; Pearson, T.J.; Readhead, A.C.S.; Wilkinson, P.N.; Biggs, A.D.; Blandford, R.D.; Fassnacht, C.D.; et al. The Cosmic Lens All-Sky Survey-I. Source selection and observations. Mon. Not. R. Astron. Soc. 2003, 341, 1-12, doi:10.1046/j.1365-8711.2003.06256.x. [CrossRef]

30. Aharonian, F.; Akhperjanian, A.G.; Aye, K.M.; Bazer-Bachi, A.R.; Beilicke, M.; Benbow, W.; Berge, D.; Berghaus, P.; Bernlöhr, K.; Boisson, C.; et al. Observations of Mkn 421 in 2004 with HESS at large zenith angles. Astron. Astrophys. 2005, 437, 95-99. doi:10.1051/0004-6361:20053050. [CrossRef] 
31. Verrecchia, F.; in't Zand, J.J.M.; Giommi, P.; Santolamazza, P.; Granata, S.; Schuurmans, J.J.; Antonelli, L.A. The BeppoSAX WFC X-ray source catalogue. Astron. Astrophys. 2007, 472, 705-713. doi:10.1051/0004-6361:20067040. [CrossRef]

32. Nieppola, E.; Tornikoski, M.; Lähteenmäki, A.; Valtaoja, E.; Hakala, T.; Hovatta, T.; Kotiranta, M.; Nummila, S.; Ojala, T.; Parviainen, M.; et al. 37 GHz Observations of a Large Sample of BL Lacertae Objects. Astron. J. 2007, 133, 1947-1953, doi:10.1086/512609. [CrossRef]

33. Healey, S.E.; Romani, R.W.; Taylor, G.B.; Sadler, E.M.; Ricci, R.; Murphy, T.; Ulvestad, J.S.; Winn, J.N. CRATES: An All-Sky Survey of Flat-Spectrum Radio Sources. Astrophys. J. Suppl. Ser. 2007, 171, 61-71, doi:10.1086/513742. [CrossRef]

34. Jackson, N.; Battye, R.A.; Browne, I.W.A.; Joshi, S.; Muxlow, T.W.B.; Wilkinson, P.N. A survey of polarization in the JVAS/CLASS flat-spectrum radio source surveys-I. The data and catalogue production. Mon. Not. R. Astron. Soc. 2007, 376, 371-377, doi:10.1111/j.1365-2966.2007.11442.x. [CrossRef]

35. Saxton, R.D.; Read, A.M.; Esquej, P.; Freyberg, M.J.; Altieri, B.; Bermejo, D. The first XMM-Newton slew survey catalogue: XMMSL1. Astron. Astrophys. 2008, 480, 611-622, doi:10.1051/0004-6361:20079193. [CrossRef]

36. Pittori, C.; Verrecchia, F.; Chen, A.W.; Bulgarelli, A.; Pellizzoni, A.; Giuliani, A.; Vercellone, S.; Longo, F.; Tavani, M.; Giommi, P. First AGILE catalog of high-confidence gamma-ray sources. Astron. Astrophys. 2009, 506, 1563-1574, doi:10.1051/0004-6361/200911783. [CrossRef]

37. Cusumano, G.; La Parola, V.; Segreto, A.; Mangano, V.; Ferrigno, C.; Maselli, A.; Romano, P.; Mineo, T.; Sbarufatti, B.; Campana, S.; et al. The Palermo Swift-BAT hard X-ray catalogue. II. Results after 39 months of sky survey. Astron. Astrophys. 2010, 510, A48, doi:10.1051/0004-6361/200811184. [CrossRef]

38. Cusumano, G.; La Parola, V.; Segreto, A.; Ferrigno, C.; Maselli, A.; Sbarufatti, B.; Romano, P.; Chincarini, G.; Giommi, P.; Masetti, N.; et al. The Palermo Swift-BAT hard X-ray catalogue. III. Results after 54 months of sky survey. Astron. Astrophys. 2010, 524, A64, doi:10.1051/0004-6361/201015249. [CrossRef]

39. Wright, E.L.; Eisenhardt, P.R.M.; Mainzer, A.K.; Ressler, M.E.; Cutri, R.M.; Jarrett, T.; Kirkpatrick, J.D.; Padgett, D.; McMillan, R.S.; Skrutskie, M.; et al. The Wide-field Infrared Survey Explorer (WISE): Mission Description and Initial On-orbit Performance. Astron. J. 2010, 140, 1868-1881, doi:10.1088/0004-6256/140/6/1868. [CrossRef]

40. Bird, A.J.; Bazzano, A.; Bassani, L.; Capitanio, F.; Fiocchi, M.; Hill, A.B.; Malizia, A.; Mc Bride, V.A.; Scaringi, S.; Sguera, V.; et al. The Fourth IBIS/ISGRI Soft Gamma-ray Survey Catalog. Astrophys. J. Suppl. Ser. 2010, 186, 1-9, doi:10.1088/0067-0049/186/1/1. [CrossRef]

41. Abdo, A.A.; Ackermann, M.; Ajello, M.; Allafort, A.; Antolini, E.; Atwood, W.B.; Axelsson, M.; Baldini, L.; Ballet, J.; Barbiellini, G.; et al. Fermi Large Area Telescope First Source Catalog. Astrophys. J. Suppl. Ser. 2010, 188, 405-436, doi:10.1088/0067-0049/188/2/405. [CrossRef]

42. Chandra, P.; Yadav, K.K.; Rannot, R.C.; Singh, K.K.; Tickoo, A.K.; Sharma, M.; Venugopal, K.; Bhat, C.K.; Bhatt, N.; Bhattacharyya, S.; et al. TeV observations of Mrk 421 with the TACTIC $\gamma$-ray telescope during 2006-8. J. Phys. G Nucl. Phys. 2010, 37, 125201, doi:10.1088/0954-3899/37/12/125201. [CrossRef]

43. Planck Collaboration; Ade, P.A.R.; Aghanim, N.; Arnaud, M.; Ashdown, M.; Aumont, J.; Baccigalupi, C.; Balbi, A.; Banday, A.J.; Barreiro, R.B.; et al. Planck early results. VII. The Early Release Compact Source Catalogue. Astron. Astrophys. 2011, 536, A7, doi:10.1051/0004-6361/201116474. [CrossRef]

44. Bartoli, B.; Bernardini, P.; Bi, X.J.; Bleve, C.; Bolognino, I.; Branchini, P.; Budano, A.; Calabrese Melcarne, A.K.; Camarri, P.; Cao, Z.; et al. Long-term Monitoring of the TeV Emission from Mrk 421 with the ARGO-YBJ Experiment. Astrophys. J. 2011, 734, 110, doi:10.1088/0004-637X/734/2/110. [CrossRef]

45. Acciari, V.A.; Aliu, E.; Arlen, T.; Aune, T.; Beilicke, M.; Benbow, W.; Boltuch, D.; Bradbury, S.M.; Buckley, J.H.; Bugaev, V.; et al. TeV and Multi-wavelength Observations of Mrk 421 in 2006-2008. Astrophys. J. 2011, 738, 25, doi:10.1088/0004-637X/738/1/25. [CrossRef]

46. Hiroi, K.; Ueda, Y.; Isobe, N.; Hayashida, M.; Eguchi, S.; Sugizaki, M.; Kawai, N.; Tsunemi, H.; Matsuoka, M.; Mihara, T.; et al. The First MAXI/GSC Catalog in the High Galactic-Latitude Sky. Publ. Astron. Soc. Jpn. 2011, 63, S677-S689, doi:10.1093/pasj/63.sp3.S677. [CrossRef]

47. Ajello, M.; Alexander, D.M.; Greiner, J.; Madejski, G.M.; Gehrels, N.; Burlon, D. The 60 Month All-sky Burst Alert Telescope Survey of Active Galactic Nucleus and the Anisotropy of nearby AGNs. Astrophys. J. 2012, 749, 21, doi:10.1088/0004-637X/749/1/21. [CrossRef] 
48. Nolan, P.L.; Abdo, A.A.; Ackermann, M.; Ajello, M.; Allafort, A.; Antolini, E.; Atwood, W.B.; Axelsson, M.; Baldini, L.; Ballet, J.; et al. Fermi Large Area Telescope Second Source Catalog. Astrophys. J. Suppl. Ser. 2012, 199, 31, doi:10.1088/0067-0049/199/2/31. [CrossRef]

49. Chandra, P.; Rannot, R.C.; Yadav, K.K.; Tickoo, A.K.; Singh, K.K.; Chanchalani, K.; Kothari, M.; Agarwal, N.K.; Goyal, A.; Goyal, H.C.; et al. TeV gamma-ray observations of Markarian 421 using TACTIC during 2009-10. J. Phys. G Nucl. Phys. 2012, 39, 045201, doi:10.1088/0954-3899/39/4/045201. [CrossRef]

50. Bartoli, B.; Bernardini, P.; Bi, X.J.; Bolognino, I.; Branchini, P.; Budano, A.; Calabrese Melcarne, A.K.; Camarri, P.; Cao, Z.; Cardarelli, R.; et al. TeV Gamma-Ray Survey of the Northern Sky Using the ARGO-YBJ Detector. Astrophys. J. 2013, 779, 27, doi:10.1088/0004-637X/779/1/27. [CrossRef]

51. Baumgartner, W.H.; Tueller, J.; Markwardt, C.B.; Skinner, G.K.; Barthelmy, S.; Mushotzky, R.F.; Evans, P.A.; Gehrels, N. The 70 Month Swift-BAT All-sky Hard X-Ray Survey. Astrophys. J. Suppl. Ser. 2013, 207, 19, doi:10.1088/0067-0049/207/2/19. [CrossRef]

52. Hiroi, K.; Ueda, Y.; Hayashida, M.; Shidatsu, M.; Sato, R.; Kawamuro, T.; Sugizaki, M.; Nakahira, S.; Serino, M.; Kawai, N.; et al. The 37 Month MAXI/GSC Source Catalog of the High Galactic-Latitude Sky. Astrophys. J. Suppl. Ser. 2013, 207, 36, doi:10.1088/0067-0049/207/2/36. [CrossRef]

53. Planck Collaboration; Ade, P.A.R.; Aghanim, N.; Argüeso, F.; Armitage-Caplan, C.; Arnaud, M.; Ashdown, M.; Atrio-Barandela, F.; Aumont, J.; Baccigalupi, C.; et al. Planck 2013 results. XXVIII. The Planck Catalogue of Compact Sources. Astron. Astrophys. 2014, 571, A28, doi:10.1051/0004-6361/201321524. [CrossRef]

54. Evans, P.A.; Osborne, J.P.; Beardmore, A.P.; Page, K.L.; Willingale, R.; Mountford, C.J.; Pagani, C.; Burrows, D.N.; Kennea, J.A.; Perri, M.; et al. 1SXPS: A Deep Swift X-Ray Telescope Point Source Catalog with Light Curves and Spectra. Astrophys. J. Suppl. Ser. 2014, 210, 8, doi:10.1088/0067-0049/210/1/8. [CrossRef]

55. Biteau, J.; Williams, D.A. The Extragalactic Background Light, the Hubble Constant, and Anomalies: Conclusions from 20 Years of TeV Gamma-ray Observations. Astrophys. J. 2015, 812, 60, doi:10.1088/0004-637X/812/1/60. [CrossRef]

56. Acero, F.; Ackermann, M.; Ajello, M.; Albert, A.; Atwood, W.B.; Axelsson, M.; Baldini, L.; Ballet, J.; Barbiellini, G.; Bastieri, D.; et al. Fermi Large Area Telescope Third Source Catalog. Astrophys. J. Suppl. Ser. 2015, 218, 23, doi:10.1088/0067-0049/218/2/23. [CrossRef]

57. Sharma, M.; Nayak, J.; Koul, M.K.; Bose, S.; Mitra, A.; Dhar, V.K.; Tickoo, A.K.; Koul, R. Validation of a new background discrimination method for the TACTIC TeV $\gamma$-ray telescope with Markarian 421 data. Nucl. Instrum. Methods Phys. Res. A 2015, 770, 42-47, doi:10.1016/j.nima.2014.10.012. [CrossRef]

58. Boller, T.; Freyberg, M.J.; Trümper, J.; Haberl, F.; Voges, W.; Nandra, K. Second ROSAT all-sky survey (2RXS) source catalogue. Astron. Astrophys. 2016, 588, A103, doi:10.1051/0004-6361/201525648. [CrossRef]

59. Rosen, S.R.; Webb, N.A.; Watson, M.G.; Ballet, J.; Barret, D.; Braito, V.; Carrera, F.J.; Ceballos, M.T.; Coriat, M.; Della Ceca, R.; et al. The XMM-Newton serendipitous survey. VII. The third XMM-Newton serendipitous source catalogue. Astron. Astrophys. 2016, 590, A1, doi:10.1051/0004-6361/201526416. [CrossRef]

60. Planck Collaboration; Ade, P.A.R.; Aghanim, N.; Argüeso, F.; Arnaud, M.; Ashdown, M.; Aumont, J.; Baccigalupi, C.; Banday, A.J.; Barreiro, R.B.; et al. Planck 2015 results. XXVI. The Second Planck Catalogue of Compact Sources. Astron. Astrophys. 2016, 594, A26, doi:10.1051/0004-6361/201526914. [CrossRef]

61. Abeysekara, A.U.; Archambault, S.; Archer, A.; Aune, T.; Barnacka, A.; Benbow, W.; Bird, R.; Biteau, J.; Buckley, J.H.; Bugaev, V.; et al. Gamma-Rays from the Quasar PKS 1441+25: Story of an Escape. Astrophys. J. Lett. 2015, 815, L22, doi:10.1088/2041-8205/815/2/L22. [CrossRef]

62. Zacharias, M.; Sitarek, J.; Dominis Prester, D.; Jankowsky, F.; Lindfors, E.; Mohamed, M.; Sanchez, D.; Terzic, T.; H. E. S. S. Collaboration; MAGIC Collaboration. The exceptional VHE gamma-ray outburst of PKS 1510-089 in May 2016. In Proceedings of the 35th International Cosmic Ray Conference (ICRC2017), Busan, Korea, 10-20 July 2017; Volume 35, p. 655.

63. Romoli, C.; Zacharias, M.; Meyer, M.; Ait Benkhali, F.; Jacholkowska, A.; Wierzcholska, A.; Jankowsky, F.; Lenain, J.P.; H. E. S. S. Collaboration. Observation of the extremely bright flare of the FSRQ 3C279 with H.E.S.S. II. In Proceedings of the 35th International Cosmic Ray Conference (ICRC2017), Busan, Korea, 10-20 July 2017; Volume 35, p. 649.

64. Shaw, M.S.; Romani, R.W.; Cotter, G.; Healey, S.E.; Michelson, P.F.; Readhead, A.C.S.; Richards, J.L.; Max-Moerbeck, W.; King, O.G.; Potter, W.J. Spectroscopy of the Largest Ever $\gamma$-Ray-selected BL Lac Sample. Astrophys. J. 2013, 764, 135, doi:10.1088/0004-637X/764/2/135. [CrossRef] 
65. Pita, S.; Goldoni, P.; Boisson, C.; Cotter, G.; Lefaucheur, J.; Lenain, J.P.; Lindfors, E.; Williams, D.A. Redshift measurement of Fermi blazars for the Cherenkov telescope array. In AIP Conference Proceedings; American Institute of Physics Conference Series; AIP Publishing: Melville, NY, USA, 2017; Volume 1792, doi:10.1063/1.4968971.

66. Abbott, B.P.; Abbott, R.; Abbott, T.D.; Acernese, F.; Ackley, K.; Adams, C.; Adams, T.; Addesso, P.; Adhikari, R.X.; Adya, V.B.; et al. Multi-messenger Observations of a Binary Neutron Star Merger. Astrophys. J. Lett. 2017, 848, L12, doi:10.3847/2041-8213/aa91c9. [CrossRef]

67. IceCube Collaboration; Aartsen, M.G.; Ackermann, M.; Adams, J.; Aguilar, J.A.; Ahlers, M.; Ahrens, M.; Al Samarai, I.; Altmann, D.; Andeen, K.; et al. Multimessenger observations of a flaring blazar coincident with high-energy neutrino IceCube-170922A. Science 2018, 361, eaat1378, doi:10.1126/science.aat1378. [CrossRef]

68. Abdo, A.A.; Ackermann, M.; Ajello, M.; Allafort, A.; Baldini, L.; Ballet, J.; Barbiellini, G.; Baring, M.G.; Bastieri, D.; Bechtol, K.; et al. Insights into the High-energy $\gamma$-ray Emission of Markarian 501 from Extensive Multifrequency Observations in the Fermi Era. Astrophys. J. 2011, 727, 129. doi:10.1088/0004-637X/727/2/129. [CrossRef]

69. Barthelmy, S.D.; Barbier, L.M.; Cummings, J.R.; Fenimore, E.E.; Gehrels, N.; Hullinger, D.; Krimm, H.A.; Markwardt, C.B.; Palmer, D.M.; Parsons, A.; et al. The Burst Alert Telescope (BAT) on the SWIFT Midex Mission. Space Sci. Rev. 2005, 120, 143-164, doi:10.1007/s11214-005-5096-3. [CrossRef]

70. Tavani, M.; Barbiellini, G.; Argan, A.; Boffelli, F.; Bulgarelli, A.; Caraveo, P.; Cattaneo, P.W.; Chen, A.W.; Cocco, V.; Costa, E.; et al. The AGILE Mission. Astron. Astrophys. 2009, 502, 995-1013.10.1051/0004-6361/200810527. [CrossRef]

71. Meegan, C.; Lichti, G.; Bhat, P.N.; Bissaldi, E.; Briggs, M.S.; Connaughton, V.; Diehl, R.; Fishman, G.; Greiner, J.; Hoover, A.S.; et al. The Fermi Gamma-ray Burst Monitor. Astrophys. J. 2009, 702, 791-804, doi:10.1088/0004-637X/702/1/791. [CrossRef]

72. Atwood, W.B.; Abdo, A.A.; Ackermann, M.; Althouse, W.; Anderson, B.; Axelsson, M.; Baldini, L.; Ballet, J.; Band, D.L.; Barbiellini, G.; et al. The Large Area Telescope on the Fermi Gamma-Ray Space Telescope Mission. Astrophys. J. 2009, 697, 1071-1102, doi:10.1088/0004-637X/697/2/1071. [CrossRef]

73. Abeysekara, A.U.; Albert, A.; Alfaro, R.; Alvarez, C.; Álvarez, J.D.; Arceo, R.; Arteaga-Velázquez, J.C.; Ayala Solares, H.A.; Barber, A.S.; Bautista-Elivar, N.; et al. Observation of the Crab Nebula with the HAWC Gamma-Ray Observatory. Astrophys. J. 2017, 843, 39, doi:10.3847/1538-4357/aa7555. [CrossRef]

74. Bühler, R.; Blandford, R. The surprising Crab pulsar and its nebula: A review. Rep. Prog. Phys. 2014, 77, 066901, doi:10.1088/0034-4885/77/6/066901. [CrossRef]

75. Abeysekara, A.U.; Albert, A.; Alfaro, R.; Alvarez, C.; Álvarez, J.D.; Arceo, R.; Arteaga-Velázquez, J.C.; Avila Rojas, D.; Ayala Solares, H.A.; Barber, A.S.; et al. Daily Monitoring of TeV Gamma-Ray Emission from Mrk 421, Mrk 501, and the Crab Nebula with HAWC. Astrophys. J. 2017, 841, 100, doi:10.3847/1538-4357/aa729e. [CrossRef]

76. Fermi-LAT Monitored Source List Light Curves. Available online: https://fermi.gsfc.nasa.gov/ssc/data/ access/lat/msl_lc/ (accessed on 15 November 2018).

77. Mirzoyan, R. Detection of very-high-energy gamma-ray emission from the FSRQ Ton 0599 with the MAGIC telescopes. Astronomer's Telegram 2017, 11061.

78. Mukherjee, R.; VERITAS Collaboration. VERITAS Detection of VHE Emission from Ton 599. Astronomer's Telegram 2017, 11075.

79. Cheung, C.C.; Gasparrini, D.; Buson, S. Fermi LAT detection of GeV flaring activity from blazar Ton 599 (4C +29.45). Astronomer's Telegram 2017, 10931.

80. Mirzoyan, R. Discovery of Very High Energy Gamma-Ray Emission From Gravitationally Lensed Blazar S3 0218+357 With the MAGIC Telescopes. Astronomer's Telegram 2014, 6349.

81. Ahnen, M.L.; Ansoldi, S.; Antonelli, L.A.; Antoranz, P.; Arcaro, C.; Babic, A.; Banerjee, B.; Bangale, P.; Barres de Almeida, U.; Barrio, J.A.; et al. Detection of very high energy gamma-ray emission from the gravitationally lensed blazar QSO B0218+357 with the MAGIC telescopes. Astron. Astrophys. 2016, 595, A98. doi:10.1051/0004-6361/201629461. [CrossRef]

82. Buson, S.; Cheung, C.C. Fermi LAT Detection of a Hard Spectrum Gamma-ray Flare from Gravitationally Lensed Blazar S3 0218+357. Astronomer's Telegram 2014, 6316. 
83. Mirzoyan, R. Discovery of Very High Energy Gamma-Ray Emission from the distant FSRQ PKS 1441+25 with the MAGIC telescopes. Astronomer's Telegram 2015, 7416.

84. Mukherjee, R. Very-high-energy gamma-ray emission from PKS 1441+25 detected with VERITAS. Astronomer's Telegram 2015, 7433.

85. Pacciani, L. Optical, X-, Gamma-ray flare of the FSRQ PKS 1441+25. Astronomer's Telegram 2015, 7402.

86. Cerruti, M.; Böttcher, M.; Chakraborty, N.; Davids, I.D.; Füßling, M.; Jankowsky, F.; Lenain, J.P.; Meyer, M.; Prokoph, H.; Wagner, S.; et al. Target of opportunity observations of blazars with H.E.S.S. In Proceedings of the 6th International Symposium on High Energy Gamma-Ray Astronomy, Heidelberg, Germany, 11-15 July 2017; American Institute of Physics Conference Series; AIP Publishing: Melville, NY, USA, 2017; Volume 1792, p. 050029, doi:10.1063/1.4968975. [CrossRef]

87. Ajello, M.; Atwood, W.B.; Baldini, L.; Ballet, J.; Barbiellini, G.; Bastieri, D.; Bellazzini, R.; Bissaldi, E.; Blandford, R.D.; Bloom, E.D.; et al. 3FHL: The Third Catalog of Hard Fermi-LAT Sources. Astrophys. J. Suppl. Ser. 2017, 232, 18, doi:10.3847/1538-4365/aa8221. [CrossRef]

88. Ackermann, M.; Ajello, M.; Albert, A.; Allafort, A.; Antolini, E.; Baldini, L.; Ballet, J.; Barbiellini, G.; Bastieri, D.; Bechtol, K.; et al. The Fermi All-sky Variability Analysis: A List of Flaring Gamma-Ray Sources and the Search for Transients in Our Galaxy. Astrophys. J. 2013, 771, 57, doi:10.1088/0004-637X/771/1/57. [CrossRef]

89. Abdollahi, S.; Ackermann, M.; Ajello, M.; Albert, A.; Baldini, L.; Ballet, J.; Barbiellini, G.; Bastieri, D.; Becerra Gonzalez, J.; Bellazzini, R.; et al. The Second Catalog of Flaring Gamma-Ray Sources from the Fermi All-sky Variability Analysis. Astrophys. J. 2017, 846, 34, doi:10.3847/1538-4357/aa8092. [CrossRef]

90. Errando, M.; Orr, M. Automated analysis of Fermi-LAT data to trigger ground-based gamma-ray observations. In Proceedings of the 32nd International Cosmic Ray Conference, Beijing, China, 11-18 August 2011; Volume 8, p. 137, doi:10.7529/ICRC2011/V08/0993. [CrossRef]

91. Lenain, J.P. FLaapLUC: A pipeline for the generation of prompt alerts on transient Fermi-LAT $\gamma$-ray sources. Astron. Comput. 2018, 22, 9-15, doi:10.1016/j.ascom.2017.11.002. [CrossRef]

92. Hauser, M.G.; Dwek, E. The Cosmic Infrared Background: Measurements and Implications. Annu. Rev. Astron. Astrophys. 2001, 39, 249-307, doi:10.1146/annurev.astro.39.1.249. [CrossRef]

93. de Naurois, M.; for the H.E.S.S. collaboration. H.E.S.S. detection of a strong VHE activity from the blazar 3C 279. Astronomer's Telegram 2018, 11239.

94. Ojha, R.; Valverd, J. Fermi-LAT detection of enhanced gamma-ray activity and hard spectrum of TXS 0506+056, located inside the IceCube-170922A error region. Astronomer's Telegram 2018, 11419.

95. Cerruti, M.; Lenain, J.P.; Prokoph, H.; for the H.E.S.S. Collaboration. H.E.S.S. discovery of very-high-energy emission from the blazar PKS 0736+017: On the location of the $\gamma$-ray emitting region in FSRQs. arXiv 2017, arXiv:1708.00658.

96. Schüssler, F.; Seglar-Arroyo, M.; Arrieta, M.; Böttcher, M.; Boisson, C.; Cerruti, M.; Chakraborty, N.; Davids, I.D.; Jankowsky, F.; Lenain, J.P.; et al. Target of Opportunity Observations of Blazars with H.E.S.S. arXiv 2017, arXiv:1708.01083.

97. Anderhub, H.; Backes, M.; Biland, A.; Boccone, V.; Braun, I.; Bretz, T.; Buß, J.; Cadoux, F.; Commichau, V.; Djambazov, L.; et al. Design and operation of FACT - the first G-APD Cherenkov telescope. J. Instrum. 2013, 8, P06008, doi:10.1088/1748-0221/8/06/P06008. [CrossRef]

98. Temme, F.; Adam, J.; Ahnen, M.; Baack, D.; Balbo, M.; Bergmann, M.; Biland, A.; Blank, M.; Bretz, T.; Brügge, K.; et al. Long-Term Monitoring of Bright Blazars in the Multi-GeV to TeV Range with FACT. Galaxies 2017, 5, 18, doi:10.3390/galaxies5010018. [CrossRef]

99. CTA Consortium; Acharya, B.S.; Agudo, I.; Samarai, I.A.; Alfaro, R.; Alfaro, J.; Alispach, C.; Alves Batista, R.; Amans, J.P.; Amato, G.; et al. Science with the Cherenkov Telescope Array. arXiv 2017, arXiv:1709.07997.

100. de Angelis, A.; Tatischeff, V.; Grenier, I.A.; McEnery, J.; Mallamaci, M.; Tavani, M.; Oberlack, U.; Hanlon, L.; Walter, R.; Argan, A.; et al. Science with e-ASTROGAM. A space mission for MeV-GeV gamma-ray astrophysics. J. High Energy Astrophys. 2018, 19, 1-106, doi:10.1016/j.jheap.2018.07.001. [CrossRef] 